\section{Kein erhöhtes Risiko für Guillain-Barré-Syndrom nach H1N1-Impfung}

Im Zusammenhang mit der Influenza-Impfungen wird gelegentlich auch über die Möglichkeit eines Guillain-Barré-Syndroms berichtet. Eine aktuelle Studie aus China zeigt die Nebenwirkungen bei der H1N1-Impfung auf.

Da a das Guillain-Barré-Syndroms eine sehr seltene Erkrankung ist, sind Untersuchungen über eventuelle Zusammenhänge zu der Grippeimpfung nur mit groß angelegten Studien möglich. Zwischen September 2009 und März 2010 wurden in China 89,6 Millionen Impfdosen von Influenza A (H1N1) verabreicht. Mögliche Nebenwirkungen wurden dann zentral gesammelt. Es wurde von insgesamt 8.067 unerwünschten Ereignissen berichtet, 6.552 (ca. 81\%) davon konnten im Zusammenhang mit der Impfung gesehen werden. 1,083 Ereignisse wurden als selten oder ernst eingestuft, davon waren knapp 97\% allergische Reaktionen.

Bei den erfassten Impflingen traten elf Fälle von Guillain-Barré-Syndrom auf, d. h. ungefähr 0,1 pro eine Million Dosen. Bei acht davon (drei waren übrigens jünger als 15 Jahre) bestand eine eindeutige Evidenz für einen Zusammenhang mit der Impfung. Die Inzidenz des GuillainBarré-Syndroms bei Kindern unter 15 Jahren war damit niedriger als die Inzi- denz vor der Studie (die Baseline betrug 1,9 Fälle bei Kindern pro 1 Million Menschen). Die Gesamtinzidenz des GuillainBarré-Syndroms in China war damit nicht höher als die Inzidenz in europäischen Ländern ( 0,6 bis 1,9 Fälle pro 1 Million Menschen). In den USA wird mit einem Fall auf 1 Million Impfdosen gerechnet.

Kommentar: Die Autoren schlussfolgern, dass ,weder ein besorgniserregendes Muster von unerwünschten Ereignissen nach Impfung gegen Influenza A (H1N1) beobachtet wurde noch ein erhöhtes Risiko für ein Guillain-Barré-Syndrom besteht".

Dr. Ulrich Mutschler

Liang X-F et al. Safety of influenza A (H1N1) vaccine in postmarketing surveillance in China. N Engl J Med 2011; 364: 638-47

\title{
Probiotika können den Impferfolg verbessern
}

Auch wer mit seinen Kindern alle Impftermine zuverlässig einhält, kann nicht sicher sein, dass diese ausreichend gegen die entsprechenden Infektionskrankheiten geschützt sind. Schuld ist eine insuffiziente Immunantwort auf die Impfung. Eine Vorbehandlung mit Probiotika könnte hier hilfreich sein.

sraelische Pädiater haben eine randomisierte Studie an 47 Kleinkindern mit dem Ziel durchgeführt, die Auswirkungen von Probiotika auf die immuno- logische Response nach dem Impfen zu eruieren. Alle Patienten der Experimentalgruppe erhielten zwei Monate vor den üblichen MMR-Impfungen orale Pro-

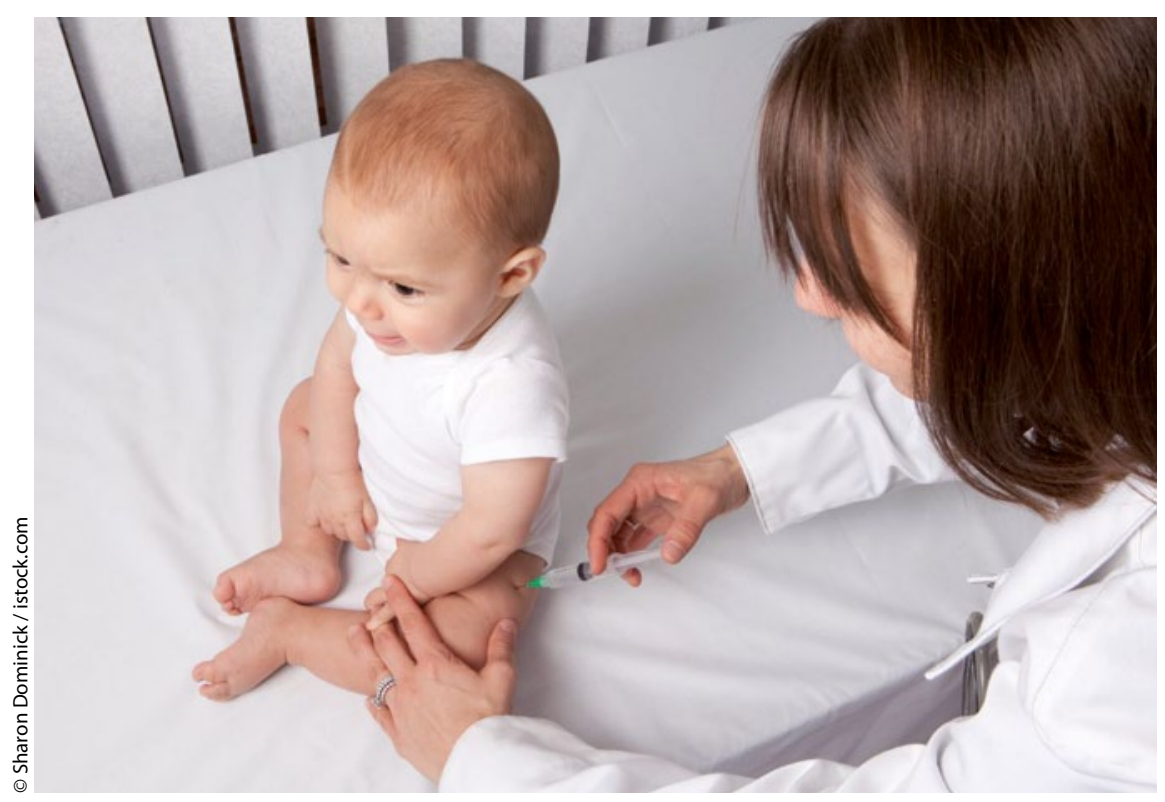

biotika. Die Kontrollgruppe erhielt Placebos. Die Therapiedauer betrug fünf Monate. Danach wurden die Antikörper bestimmt.

Der Prozentsatz der Kleinkinder, der keine ausreichenden Antikörpertiter aufwies, war $17 \%$ in der Placebo- und $8 \%$ in der Probiotikagruppe. Die Rate der Impfreaktionen war tendenziell in der Probiotikagruppe niedriger als unter Placebo.

Kommentar: Probiotika modifizieren immunologische Reaktionen. Es war daher folgerichtig zu prüfen, ob diese Mittel den Impferfolg verbessern können. Die Studie zeigt, dass Probiotika hier erfolgreich sind. Allerdings sind die Differenzen nur grenzwertig signifikant, wohl auch wegen der geringen Probandenzahl. Die Ergebnisse bedürfen daher einer unabhängigen Überprüfung.

Prof. Dr. Edzard Ernst

Youngster l et al. Probiotics and the immunological response to infant vaccinations: a prospective, placebo controlled pilot study. Arch Dis Child 2011; 96: 345-9

Mit einer begleitenden Probiotikaeinnahme steigt die Chance auf ausreichende Antikörpertiter. 